\title{
Does the Brain Think?
}

\section{Erlend Winderen Finke Owesen ${ }^{1}$ (D)}

Accepted: 17 January 2022

(c) The Author(s) 2022

\begin{abstract}
It is common in cognitive science to ascribe psychological predicates to the brain, i.e. to assert that the brain sees, feels, thinks, etc. This has prompted philosophical debate. According to the Nonsense View, the relevant locutions of cognitive scientists are nonsensical or false (Bennett and Hacker 2003, 2007). According to the Literal View, they are literal truths and report the psychological properties of brains (Dennett 2007; Crane 2015; Figdor 2018). In this paper, I propose the Synecdoche View, according to which cognitive scientists' locutions are figurative, with 'brain' referring to the human being, such that 'the brain thinks' reports the thinking of the human being, not the thinking of the brain. I compare this view to the dominant views in the literature and argue that it is a plausible alternative. One consequence of this is that there is no reason to believe that the locutions of cognitive scientists indicate empirical support for the claim that brains possess psychological properties.
\end{abstract}

\section{Introduction}

Cognitive neuroscientists and psychologists often ascribe psychological predicates to the brain. Here are some examples discussed by philosophers (I have put psychological predicates in bold):

What you see is not what is really there; it is what your brain believes is there [...] Your brain makes the best interpretation it can according to its previous experience and the limited and ambiguous information provided by your eyes (Crick 1994: 31, quoted by Bennett and Hacker 2003: 68).

[...] our brains can often decide well, in seconds, or minutes, depending on the time frame we set as appropriate for the goal we want to achieve, and if they can do so, they must do the marvellous job with more than just pure reason (Damasio 1996: 172-173, quoted by Bennett and Hacker 2003: 69).

Erlend Winderen Finke Owesen ewfo2@cam.ac.uk

1 Department of History and Philosophy of Science, University of Cambridge, Cambridge, UK 
[T] he light of the Sun always comes from above. This means that concave objects will be light at the top and dark at the bottom, while convex objects will be light at the bottom and dark at the top. Our brain has this simple rule built into its wiring. It uses this rule to decide whether an object is concave or convex [...] (Frith 2007: 128, quoted by Crane 2015: 253).

If we are to take these locutions at face value, the brain believes, makes interpretations, has experiences, and decides.

Despite the widespread practice of cognitive scientists to ascribe psychological predicates to the brain, whether and how we can make sense of these locutions has in recent years been a matter of controversy among philosophers. In this debate, Bennett and Hacker (2003, 2007) defend what I (following Figdor 2018) call the Nonsense View, according to which cognitive scientists' ascriptions of psychological predicates to the brain are nonsensical or false. They believe that psychological predicates apply literally only to human beings or other animals as a whole and that brain behaviour - such as activity detected in an fMRI scan - is not a legitimate ground for ascribing psychological predicates to the brain. Against this, Dennett (2007), Crane (2015) and Figdor (2018) defend what I call the Literal View, according to which the locutions of cognitive scientists are literal truths and report the psychological properties of brains. On their view, brain behaviour is a legitimate ground for ascribing psychological predicates to the brain. ${ }^{1}$

In what follows, I shall propose a novel view that accommodates aspects from both these views. According to this view, which I call the Synecdoche View, we can make sense of the scientific practice by understanding the word 'brain' figuratively, namely as a synecdoche (a word for a part referring to a whole, or vice versa) referring to the human being. Just like I can use 'my new set of wheels' to refer to my new car, so cognitive scientists use 'brain' to refer to the human being. On this view, 'The brain thinks' reports the thinking of the human being, not the thinking of the brain. My main thesis is a modest one: that the Synecdoche View is a plausible alternative. I do not make the further claim that it is the best alternative. Still, the modest claim has interesting consequences. One consequence is that, contrary to what defenders of both the Nonsense View and the Literal View assume, there is no reason to take the controversial locutions literally. Another consequence is that, contrary to what e.g. Figdor claims (Figdor 2018: 5-6, 61), there is no reason to believe that these locutions indicate empirical support for the claim that brains possess psychological properties, or that scientists have discovered that psychological predicates extend more widely than what is often assumed. Before laying out the structure of the paper, three clarifications are needed.

Firstly, the debate with which this paper is concerned is not about speakermeaning. Speaker-meaning is what a speaker intends to communicate with a sentence or refer to by a word, and one can investigate what it is by asking the speaker what he or she means by the sentence or word he or she utters. If the debate were about speaker-meaning, then the above-mentioned philosophical theories would be

\footnotetext{
1 As I explain in footnote 10, Figdor's view may be interpreted in a different way.
} 
pointless, as one should rather ask the relevant scientists what they mean when they say things like 'The brain thinks'. ${ }^{2}$ But asking scientists what they mean would not be relevant for the debate with which this paper is concerned, since this debate is about the semantic meaning (i.e. truth-conditions) of the controversial locutions, not the speaker-meaning. The semantic meaning of the controversial locutions is less straightforward to investigate than the speaker-meaning, because, as will become clear, it involves considerations about e.g. what we are, the ideal of charitable interpretation, grounds for assertion, etc. Scientists are of course free to contribute to this debate, but that would involve engaging with considerations to be discussed in this paper, it would be less helpful to unreflectively report speaker-meaning. Analogously, when philosophers discuss whether the language of physical theories refer to unobservable entities, they are not interested in what the scientists formulating those theories intend to communicate (speaker-meaning), but what the truth-conditions of those theories are. Just as it would be minimally interesting for the scientific realism debate to just ask scientists whether they intend to refer to unobservable entities, so it would be minimally interesting for the debate with which this paper is concerned to just ask scientists what they mean when they ascribe psychological predicates to the brain.

Secondly, the semantic question with which this paper is concerned must be separated from the metaphysical question of whether the brain possesses psychological properties, i.e. whether it thinks etc. Still, these questions are related, since if the relevant locutions of cognitive scientists should be taken as literal truths, then these locutions indicate empirical support for the claim that brains possess psychological properties (Figdor 2018: 5-6, 61). At least if we assume the commonly accepted epistemic authority of science. If these locutions should not be taken as literal truths, however, then they do not indicate such empirical support. In other words, an answer to the semantic question determines whether these locutions indicate empirical support for a positive answer to the metaphysical question.

Thirdly, even though I follow defenders of the Nonsense View and the Literal View in appealing to the literal/non-figurative vs. non-literal/figurative distinction when formulating the semantic views, these views do not rely on that distinction, and they could be formulated in terms of truth-conditions only. The Nonsense View is the view that the truth-conditions of 'The brain thinks' are never satisfied in the relevant cognitive scientific context. The Synecdoche View is the view that 'The brain thinks' inherits its truth-conditions from 'The human being thinks' (in the relevant context). And the Literal View is the view that the truth-conditions of 'The brain thinks' are satisfied independently of whether the truth-conditions for 'The human being thinks' are satisfied (in the relevant context). So even though the literal/non-figurative vs. non-literal/figurative distinction is controversial (Wilson and

\footnotetext{
2 Though it is not certain that one would get uniform answers. Cognitive scientists who discuss the relevant locutions disagree about how they should be interpreted (see Smythies 2009; Bennett 2007), and those who do not discuss this may not have thought much about the issue.
} 
Sperber 2002) that need not affect the debate with which this paper is concerned, and I shall continue to use terms like 'literal' and 'figurative'. ${ }^{3}$

$\S 2$ introduces the Nonsense View and reconstructs Bennett and Hacker's argument for that view, which provides a useful background for the rest of the paper. $\S 3$ introduces the Literal View. $\$ 4$ identifies five assumptions made by defenders of both the Nonsense View and the Literal View, which is important both in order to understand the debate and to identify alternatives to the Nonsense View and the Literal View. $\$ 5$ argues that one of these assumptions - that cognitive scientists ascribe psychological predicates to the brain on the basis of the behaviour of the brain and not on the basis of the behaviour of the human being - is false. This opens for the Synecdoche View, which I develop in $\$ 6$. $\$ 7$ compares the Synecdoche View to the alternative views and argues that while it is preferable to the Nonsense View, the Literal View and the Synecdoche View are on par in the sense that there is no reason to prefer one rather than the other. $\S 8$ concludes that the Synecdoche View is a plausible alternative and draws out two consequences.

\section{The Nonsense View}

According to (the standard formulation of) the Nonsense View, the locutions of cognitive scientists make no sense. This view is defended by Bennett and Hacker (2003, 2007) and their argument for the view is this. ${ }^{4}$

(P1) Only if an entity X can, under normal circumstances, behave in a way that satisfies criteria for the application of psychological predicates, do literal ascriptions of psychological predicates to $\mathrm{X}$ make sense.

(P2) It is only whole animals like human beings, not brains, that can, under normal circumstances, behave in a way that satisfies criteria for the application of psychological predicates.

(P3) Cognitive scientists make literal ascriptions of psychological predicates to the brain.

(C) Therefore, cognitive scientists' ascriptions of psychological predicates to the brain do not make sense.

\footnotetext{
3 A further terminological point is that one could have phrased the debate in terms of a "personal/subpersonal' distinction. One could then say that defenders of the Nonsense View and the Literal View claim that the controversial locutions involve psychological attributions at the 'sub-personal level' (but they disagree about the truth-value of these attributions), while defenders of the Synecdoche View claim that the controversial locutions actually involve psychological attributions at the 'personal level'. Nothing substantive turns on what terminology one uses, but I shall avoid this terminology, since it is associated with quite different things for different authors (see Drayson 2014 for an overview, and Dennett 1975 for an early use of the terminology).

${ }^{4}$ Bennett and Hacker do not present a recognizable deductive argument, leading Dennett to claim that their view is held 'without argument' (Bennett and Hacker 2007: 86). However, I believe that a charitable interpretation of what they say and some background from Hacker's other publications suggests the argument that I outline here.
} 
By 'criteria' Bennett and Hacker mean the behavioural grounds on which we typically ascribe psychological predicates to animals and human beings. These include crying or limping for the ascription of pain, following moving objects with one's eyes or squinting in strong light for the ascription of seeing, and reaching out for things and trying to get them for the ascription of wanting. Similarly with other psychological predicates (Bennett and Hacker 2003: 81-83).

The motivation for (P1) is the claim that criteria are part of the meaning of psychological predicates. This claim is based on (a) a Wittgensteinian view of meaning, according to which the meaning of a word is determined by explanations of meaning, which again are determined by use ${ }^{5}$, and (b) the claim that to specify the criteria for the application of predicates such as 'being in pain', 'seeing', or 'wanting' is to give explanations of what these predicates mean (Hacker 1990: 552). Of course, Bennett and Hacker's claim is not that criteria have to be satisfied by an entity X at time $t$ if the ascription of a psychological predicate to $\mathrm{X}$ at $t$ is to make sense. It obviously makes sense to ascribe wanting to a person at $t$ even though the person does not exhibit any behavioural characteristics of wanting at $t$. That is why (P1) says that literal ascriptions of psychological predicates to $\mathrm{X}$ only make sense if $\mathrm{X}$ can behave in a way that satisfies criteria for the application of psychological predicates (Bennett and Hacker 2003: 112, 82n35, Bennett 2007: 135). The qualification 'under normal circumstances' is necessary because there are some people who cannot behave in a way that satisfies criteria but to whom it still makes sense to ascribe psychological predicates, namely paralysed people. A paralysed person would be able to behave in a way that satisfies criteria 'under normal circumstances', i.e. if he or she were not paralysed. ${ }^{6}$

The motivation for (P2) is intuition. According to Bennett and Hacker, it is intuitively obvious that brains cannot - under any circumstances - cry, limp, follow moving objects with their eyes, squint in strong light, or reach out for and try to get things (Bennett and Hacker 2003: 83, Bennett and Hacker 2007: 141, 149). On their view, it is only human beings (or animals), not brains, that can behave in a way that satisfies criteria for the application of psychological predicates.

The motivation for (P3) is the existence of passages like the ones quoted in $\S 1$, where cognitive scientists apparently make literal ascriptions of psychological predicates to the brain (Bennett and Hacker 2003: 68-81). Given Bennett and Hacker's belief that cognitive scientists' ascriptions of psychological predicates to the brain are nonsensical, one may think that they cannot accept (P3), because one may think the ascription of psychological predicates to the brain cannot be both literal and nonsensical. But Bennett and Hacker's notion of nonsense is not that of unrecognizable gibberish. Rather, what they mean by 'nonsense' is a use of words that does not conform to explanations of meaning, which they take to be rules for the use of words.

\footnotetext{
5 See Baker and Hacker (2005: 29-43, 129-158) and Hacker (2013: 123-127) for more on this view.

${ }^{6}$ Bennett and Hacker do not themselves include the qualifier 'under normal circumstances', which made critics point to paralysed people as a counterexample to Bennett and Hacker's claim that it only makes sense to ascribe psychological predicates to an entity that can behave in a way that satisfies criteria (Searle 2007: 105). But including the qualifier gets Bennett and Hacker around this worry.
} 
Given this notion of nonsense, it seems possible that one can ascribe psychological predicates to the brain - i.e. use 'brain' as the grammatical subject of psychological verbs - all with a literal sense, even though such ascriptions are (according to Bennett and Hacker) nonsensical.

From (P1), (P2) and (P3), Bennett and Hacker conclude that cognitive scientists are committing a 'mereological fallacy': they ascribe predicates to a part of an animal which it would only make sense to ascribe to the whole animal (Bennett and Hacker 2003: 73, Bennett and Hacker 2007: 132).

This is both how Bennett and Hacker themselves and their opponents (Dennett 2007; Crane 2015; Figdor 2018) understand the Nonsense View. Nevertheless, I think there is an alternative characterization of the position (at least a closely related one) that does not invoke Bennett and Hacker's particular notion of nonsense. In more standard terminology, their view is really the view that the controversial locutions of cognitive scientists are false. And the argument is this:

(P1) Only if an entity X can, under normal circumstances, behave in a way that satisfies criteria for the application of psychological predicates, can the truth-conditions for literal ascriptions of psychological predicates to $\mathrm{X}$ be satisfied.

(P2) It is only whole animals like human beings, not brains, that can, under normal circumstances, behave in a way that satisfies criteria for the application of psychological predicates.

(P3) Cognitive scientists make literal ascriptions of psychological predicates to the brain.

(C) Therefore, cognitive scientists' ascriptions of psychological predicates to the brain do not satisfy the truth-conditions for literal ascriptions of psychological predicates to the brain.

For present purposes, we can take these alternative formulations of Bennett and Hacker's view as equivalent. But I shall refer to their position as the 'Nonsense View' and their argument as the 'Nonsense Argument'. In \$3 I introduce the Literal View, which denies the conjunction of (P1) and (P2) but accepts (P3). In $\$ 6$ I develop the Synecdoche View, which is agnostic about (P1) and (P2) but denies (P3).

\section{The Literal View}

According to the Literal View, the controversial locutions of cognitive scientists report literal truths about the psychological properties of brains. There are interesting differences in Dennett's, Crane's and Figdor's formulations of this view, but the essential claim that matters in the present context is the same, namely that there are behavioural similarities between brains and humans that warrant the extension of psychological predicates to brains (Dennett 2007: 78, Crane 2015: 259-262, Figdor 2018: 96).

What behavioural similarities are there? Defenders of the Literal View are not explicit about this. But Crane (2015) and Figdor (2018) claim that the similarities 
in question can be captured by mathematical models (Crane 2015: 262, Figdor 2018: 96), which means that the similarities in question need not be what one would intuitively think of as behavioural similarities. Even though the brain may not cry, squint in strong light and try to get things, there may be other 'quantitative' similarities between the brain and the human being that warrant ascribing pain, seeing and wanting to the brain. Consequently, defenders of the Literal View can maintain that the locutions of cognitive scientists make sense and express truths, which, after all, a charitable interpretation of the linguistic practice of a successful science should aim for.

Even though defenders of the Literal View disagree with the conclusion of the Nonsense Argument, they agree with Bennett and Hacker that cognitive scientists use the word 'brain' and psychological predicates with a literal sense, i.e. they accept (P3). Given this, they must deny either (P1) or (P2) or both.

If they take (P1) to be false, their objection to the Nonsense Argument is that one can ascribe psychological predicates to an entity (a brain) that cannot behave in a way that satisfies behavioural criteria (in Bennett and Hacker's sense), because it behaves in a way sufficiently similar to something satisfying such criteria. If they take (P2) to be false, their objection is that brain behaviour is sufficiently similar to human behaviour to satisfy behavioural criteria. The difference between these alternatives is just a matter of what one calls 'criteria' - i.e. whether one accepts Bennett and Hacker's restricted notion of criteria or whether one adopts a less restricted notion where behavioural similarity captured in mathematical models counts as satisfying criteria. This is merely a verbal difference and is not important for understanding the Literal View. What is important is that defenders of the view reject the conjunction of (P1) and (P2) because they think there is sufficient similarity between brains and human beings.

The fundamental disagreement between the Nonsense View and the Literal View then, concerns whether brain behaviour is a legitimate ground for ascribing psychological predicates to the brain. Defenders of the Nonsense View hold that brain behaviour is not a legitimate ground, while defenders of the Literal View hold that brain behaviour is a legitimate ground. In $\$ 5$, I argue that this dispute about brain behaviour is based on a misunderstanding of the scientific practice, because the locutions of cognitive scientists are not based on the observation of (or a mathematical model of) brain behaviour. This makes the dispute about brain behaviour orthogonal to the semantic question with which the debate is concerned.

\section{Five Assumptions}

Before I can argue that the controversial locutions of cognitive scientists are not based on the observation of (or a mathematical model of) brain behaviour, I must first make explicit five assumptions, which are implicit in the debate between defenders of the Nonsense View and the Literal View. This is important both in order to understand the debate, and to identify alternatives to the Nonsense View and the Literal View. 
The first assumption is this:

(1) We are human beings and not brains.

If defenders of the Nonsense View and the Literal View held that we are brains (like Parfit 2012), then the Nonsense View would be the view that it makes no sense (or is false) to say that beings like us think, while the Literal View would be the view that it is literally true to say that beings like us think. But it would have been surprising if the disagreement between these views concerned whether beings like us think in lack of any statement that that is what the debate is about. Thus, it is plausible that defenders of both views assume (1).

The second assumption is this:

(2) We possess psychological properties.

Given (1), it is plausible that defenders of the Nonsense View and the Literal View assume (2). If they denied (2) and held that we do not think, then defenders of the Nonsense View would be committed to the claim that neither we human beings, nor brains, think, and defenders of the Literal View would be committed to the claim that only brains think, while we human beings do not. But it would have been surprising if they had thought that these claims were true without making that explicit in the context of the debate about the controversial locutions of cognitive scientists. Thus, it is plausible that defenders of both views assume (2).

The third assumption is this:

(3) Cognitive scientists use psychological predicates with a literal sense.

That defenders of the Nonsense View assume (3) is clear from the fact that this view is motived by the claim that the brain does not satisfy criteria (in Bennett and Hacker's sense) for the ascription of thinking - in the sense in which human beings think - which is a literal sense. For it would not matter whether the brain does not satisfy criteria for the ascription of thinking in a literal sense if the sense of 'thinking' relevant to the locutions of cognitive scientists was not literal but a non-literal figure of speech.

A similar point applies to the Literal View, since this view is motivated by the claim that the brain behaves in a way that is sufficiently similar to the behaviour on the basis of which one ascribes thinking - in a literal sense - to the human being. For it would not matter whether the brain behaves in a way that is sufficiently similar to behaviour that grounds the ascription of thinking in a literal sense if the sense of 'thinking' relevant to the locutions of cognitive scientists was not literal but a non-literal figure of speech. 
In other words, if, contrary to what (3) says, cognitive scientists used psychological predicates with a figurative sense, then the Nonsense View and the Literal View would have no relevance for the semantics of the controversial locutions of cognitive scientists. Thus, defenders of both views plausibly assume (3).

The fourth assumption is this:

(4) Cognitive scientists use the word 'brain' with the literal sense.

If cognitive scientists used the word 'brain' with a figurative sense, then it would not matter that the brain - in the literal sense - does not satisfy criteria for the ascription of psychological predicates, which is the claim that motivates the Nonsense View. It would also not matter that the brain - in the literal sense - behaves similarly to the human being, which is the claim that motivates the Literal View. So if, contrary to what (4) says, the defenders of these views held that cognitive scientists do not use 'brain' with the literal sense, then again, neither of these views would have any relevance for the semantics of the locutions of cognitive scientists. Thus, defenders of both views plausibly assume (4).

The fifth assumption is this:

(5) Cognitive scientists ascribe psychological predicates to the brain on the basis of the behaviour of the brain and not on the basis of the behaviour of the human being.

Given the dispute about whether brain behaviour is a legitimate ground for ascribing psychological predicates to the brain, it is clear that defenders of both the Nonsense View and the Literal View assume (5). It is also clear from specific passages. For example, Bennett and Hacker say that 'brain activity detected by PET or fMRI [...] does not show that the brain is thinking' (Bennett and Hacker 2003: 83) and that ' $[\mathrm{t}]$ he constitutive grounds upon which competent speakers of our language apply such [psychological] expressions to animals and human beings, namely, what they say and do, cannot be satisfied by a brain or its parts' (Bennett 2007: 149). And Crane (2015) - commenting on ascribing inference to the brain - says that '[i]nferences relate propositions, so the claim is that what is going on in the brain resembles a relationship between propositions' (Crane 2015: 262). It is difficult to make sense of these passages without appeal to (5), so defenders of both views plausibly assume (5).

In the remainder of this paper, I shall follow defenders of the Nonsense View and the Literal View in assuming (1), (2), and (3), and I shall not provide any argument for these assumptions. But in $\$ 5 \mathrm{I}$ argue that (5) is false. This makes (4) questionable, which I argue in $§ \S 6-7$. 


\section{Why Do Cognitive Scientists Say 'The Brain Thinks'?}

Contrary to the assumption of defenders of the Nonsense View and the Literal View, cognitive scientists $d o$ ascribe psychological predicates to the brain on the basis of the behaviour of the human being.

Take again the passage from Frith quoted in $\$ 1$ (and by Crane 2015: 253):

[T] he light of the Sun always comes from above. This means that concave objects will be light at the top and dark at the bottom, while convex objects will be light at the bottom and dark at the top. Our brain has this simple rule built into its wiring. It uses this rule to decide whether an object is concave or convex $[\ldots]$

The passage continues:

[...] which you can test by looking at the figure below. [...] We interpret the spots as concave and convex because the shading suggests there are shadows caused by light coming from above (Frith 2007: 128).

On the assumption of defenders of the Nonsense View and the Literal View, this should be taken as ascribing a decision to the brain on the basis of (a mathematical model of) brain behaviour and not on the basis of human behaviour. But the above passage suggests otherwise. Frith does not ascribe a decision to the brain on the basis of an fMRI scan or anything like that, but rather on the basis of the behaviour of the whole human being. When Frith says that you can test how the brain decides whether an object is concave or convex by looking at the figures in his book, 'you' refers to the reader who (given the assumption that we are human beings) is a human being. So it is the behaviour of human beings, how 'we' report seeing concave or convex objects, which is the ground for asserting that the 'brain' decides whether an object is concave or convex.

The same applies to the quoted passages of Damasio (1996) and Crick (1994). Here again is the passage from Damasio (quoted by Bennett and Hacker 2003: 69):

[...] our brains can often decide well, in seconds, or minutes, depending on the time frame we set as appropriate for the goal we want to achieve, and if they can do so, they must do the marvellous job with more than just pure reason (Damasio 1996: 172-173).

This claim is not based on the observation of (or a mathematical model of) brain behaviour. Rather, it is based on the observation of the behaviour of human beings. For the context of this quote is Damasio criticizing a rationalistic model of decisionmaking, according to which all decisions are performed by calculating costs and benefits of different courses of action. Shortly before the above quote, he says:

Now, let me submit that if this [rationalistic] strategy is the only one you have available, rationality, as described above, is not going to work. At best, your decision will take an inordinately long time, far more than acceptable if you 
are to get anything else done that day. At worst, you may not even end up with a decision at all because you will get lost in the byways of your calculation (Damasio 1996: 172).

Here 'you' refers to the reader, who, given the assumption that we are human beings, is a human being. It is the fact that you, as well as most other people, take courses of action without spending the entire day calculating costs and benefits, which is the evidence that 'our brains can often decide well is seconds, or minutes'. So it is human behaviour which serves as the evidence for the claim about 'brain' decisions.

Here again is the passage from Crick (quoted by Bennett and Hacker 2003: 68):

What you see is not what is really there; it is what your brain believes is there [...] Your brain makes the best interpretation it can according to its previous experience and the limited and ambiguous information provided by your eyes (Crick 1994: 31).

These claims are also not based on the observation of (or a mathematical model of) brain behaviour, but rather on the observation of the behaviour of human beings. For these claims are based on human beings reporting seeing one determinate thing, despite being presented with ambiguous visual stimuli. In one of Crick's examples:

[...] suppose you are looking at a photograph or a realistic painting of some scene. Moving your head or using both eyes merely tells you that, in reality, the photograph or picture is flat. Yet in most cases you still see what is represented in the picture in three dimensions (Crick 1994: 29).

Again, 'you' refers to the reader, who, given the assumption that we are human beings, is a human being. It is the fact that human beings report (among other things) seeing three dimensions in a two-dimensional object which is the ground for saying that 'What you see is not what is really there; it is what your brain believes is there' etc. So the ground for which psychological predicates are ascribed to the 'brain' in this context is human behaviour, contrary to the assumption of defenders of the Nonsense View and the Literal View. The same is true of other examples discussed in the debate (e.g. Sperry 1974: 11, Gazzaniga 1995: 225-226).

The fact that cognitive scientists $d o$ ascribe psychological predicates to the brain on the basis of the behaviour of the human being, contrary to what defenders of the Nonsense View and the Literal View assume, has two consequences. The first is that the dispute about whether brain behaviour is a legitimate ground for ascribing psychological predicates to the brain - i.e. the dispute about (P1) and (P2) of the Nonsense Argument - is orthogonal to the semantic question with which the debate is concerned. It does not matter for the semantics of the relevant locutions of cognitive scientists whether brain behaviour is a legitimate ground for ascribing psychological predicates to the brain, since these locutions are not based on the observation of (or a mathematical model of) brain behaviour. The second consequence is that an alternative semantics of the locutions of cognitive scientists becomes available: the Synecdoche View. 


\section{The Synecdoche View}

The lesson of $\S 5$ was that cognitive scientists ascribe psychological predicates to the brain on the basis of the behaviour of the human being. But the behaviour of the human being is not uniquely a ground for ascribing psychological predicates to the brain, it is simultaneously a ground for ascribing such predicates to the human being. In the example of Frith, how human beings report seeing concave or convex objects is not just a ground for saying that the brain decides whether an object is concave or convex, but simultaneously a ground for saying that the human being decides whether an object is concave or convex. In the example of Damasio, how human beings take courses of action without spending the entire day calculating costs and benefits is not just a ground for saying that the brain decides in seconds or minutes, but simultaneously a ground for saying that the human being decides in seconds or minutes. And in the example of Crick, how human beings report seeing one determinate thing, despite being presented with ambiguous visual stimuli, is not just a ground for saying that the brain's beliefs and interpretations determine what you see. It is simultaneously a ground for saying that your beliefs and interpretations - considered as the beliefs and interpretations of the human being - determine what you see.

Given that the grounds for ascribing psychological predicates to the brain are simultaneously grounds for ascribing psychological predicates to the human being, and the three first assumptions identified in $\$ 4-$ that (1) we are human beings and not brains, (2) we possess psychological properties, and (3) cognitive scientists use psychological predicates with a literal sense - I propose a novel view: the Synecdoche View. Synecdoche is a subclass of metonymy where a term for a part refers to a whole (or vice versa), like 'my new set of wheels' refers to my new car. On the Synecdoche View, we can take the controversial locutions of cognitive scientists as non-literal figures of speech, namely synecdoches in which a term for a part ('brain') refers to a whole (human being). In the examples of Frith and Damasio, the ascription of deciding to the brain reports the deciding of the human being. And in the example of Crick, the ascription of beliefs, interpretations and experiences to the brain reports the beliefs, interpretations and experiences of the human being.

Of course, it may be that the scientists themselves implicitly adopt a semantic view like the Literal View and believe that 'brain' refers to the brain. But as noted in $\S 1$, the Synecdoche View is not about speaker-meaning, so if scientists implicitly adopt the Literal View, then the speaker-meaning of 'The brain thinks' diverges from the semantic meaning (i.e. truth-condition) of that sentence. But that is no objection to the Synecdoche View, because that view is only a claim about semantic meaning, not speaker-meaning.

One important fact about synecdoches is that they often exploit associations of parts and wholes (Lakoff and Johnson 1980: 35-40). Given that psychological properties are closely associated with the brain we can see what might motivate using 'brain' as a synecdoche for 'human being'. Using 'brain' synecdochically in talk of psychological properties indicates the functional relevance of the brain for instantiating such properties. It would have been arbitrary to use e.g. 'lung' instead of 'brain' just as it would have been arbitrary to use 'doors' instead of 'wheels' in 'my new set of wheels', given that wheels are more closely associated with the function of a car than doors are. 
Of course, this does not prevent anyone from using 'lung' and 'doors' as synecdoches for human being and car respectively, since one is free to use words however one likes. The point, however, is that it would (in normal contexts) be arbitrary to use 'lung' and 'doors' that way, while the synecdochical use of 'brain' and 'wheels' might be motivated by the functional relevance of brains and wheels.

Synecdoches are figures of speech, so according to the Synecdoche View, scientists' ascription of psychological predicates to the brain is a figurative way of saying what human beings literally do. The psychological predicates can be understood literally, but the use of 'brain' is figurative and refers to the human being. ${ }^{7}$ This does not imply that every time cognitive scientists use the word 'brain' they are referring to a human being and not a brain. For example, when a scientist says that 'our fMRI scan detected activity in the brain', then 'brain' refers to the brain. But in the controversial locutions under discussion, it is a live possibility that 'brain' refers to the human being.

It should be noted that sometimes the human being is not conscious of the psychological states that cognitive scientists ascribe to the brain, in the sense that the human being does not have any higher-order awareness of the relevant psychological states. The example of Frith may be one example, since one may not be conscious of one's deciding that an object is concave or convex, one is only conscious of seeing the object as concave or convex. The same may be true of the example of Crick, since one may not be conscious of one's beliefs and interpretations that determine what one sees, one is only conscious of seeing something a determinate way.

In fact, the same point seems to apply to most ascriptions of psychological predicates to the brain in the predictive processing literature - where the brain is said to predict, guess, estimate or expect the causes of sensory input using Bayesian models. Consider the following passage from Körding and Wolpert (2004):

the central nervous system [...] employs probabilistic models during sensorimotor learning (Körding and Wolpert 2004: 244).

This hypothesis is supposed to explain how tennis players are able to estimate the velocity of a tennis ball and move accordingly. So like in the examples above, the ascription of a psychological predicate to the brain (or central nervous system) is based on the behaviour of the human being (i.e. the tennis player moving according to the ball's velocity), and 'the central nervous system' may be a synecdoche for the human being. But it is clear that the human being does not consciously estimate anything using Bayesian models. ${ }^{8}$

\footnotetext{
7 Because synecdoches and other metonyms are figures of speech, it is hard to understand Machamer and Sytsma's (2009) view that the ascription of psychological predicates to the brain is both a form of metonymy where a part refers to a whole and a literal extension of psychological predicates to describe brain behaviour (Machamer and Sytsma 2009: 358-360). One and the same scientist cannot use the word 'brain' both literally - referring to the brain, and metonymically - referring to the human being - at one and the same time.

8 This interpretation is in agreement with several things Körding and Wolpert say themselves. For example, they say that 'subjects implicitly use Bayesian statistics' (Körding and Wolpert 2004: 246). Here 'subjects' plausibly refers to human beings, and 'implicitly' plausibly refers to the fact that subjects lack a higher-order awareness of their Bayesian estimation.
} 
The claim that the psychological states in question are unconscious is consistent with my assumption that the relevant psychological predicates are literal. Even if conscious deciding is different from unconscious deciding, it does not follow that 'decide' is literal only in the context of conscious deciding, while being a non-literal figure of speech in the context of unconscious deciding. Conscious and unconscious deciding can both be deciding literally speaking, it is just that one is accompanied by higher-order awareness while the other is not. And both kinds of psychological states can be ascribed to the human being. So even though human beings are not conscious of the psychological states referred to by the controversial locutions of cognitive scientists, the Synecdoche View can still accommodate the relevant locutions. At least as long as the grounds for ascribing psychological predicates to the brain are simultaneously grounds for ascribing the same predicates to the human being.

The latter qualification points to an important point about the scope of the Synecdoche View. The view applies to the locutions of cognitive scientists documented by philosophers, such as those quoted in $\S 1$, which prompted the philosophical debate. These locutions all involve ascriptions of psychological predicates to the brain made on grounds that are simultaneously grounds for ascribing those predicates to the human being. Perhaps there are also examples of cognitive scientists ascribing psychological predicates to the brain on grounds that are uniquely grounds for ascribing those predicates to the brain. If so, the Synecdoche View does not apply to these examples. But no such examples have been documented in the debate. The locutions discussed above are not cherry-picked by me, they are examples discussed by proponents of the Nonsense View and the Literal View. ${ }^{9}$

One may think that the restricted scope of the Synecdoche View makes it a less powerful view compared to a view that offers a unified account - regardless of whether grounds for assertion are uniquely grounds for asserting that the brain thinks or simultaneously grounds for asserting that the human being thinks. Perhaps the Nonsense View and the Literal View offer such unified accounts. But it is plausible that grounds for assertion matter for semantics. 'My new set of wheels was expensive' should be interpreted literally when asserted on the basis of the new wheels I have bought to replace the old ones of the old car, and it should be interpreted synecdochically when asserted on the basis of the new car I have bought. It would be strange to insist on one single semantics for the sake of 'unity'.

\footnotetext{
${ }^{9}$ One set of locutions that demand a separate discussion, which I shall therefore only mention briefly here, is that of psychological attributions to the hemispheres of so-called 'split-brain' patients (e.g. Sperry 1974: 11, Gazzaniga 1995: 225-226). In these cases, scientists say e.g. that the left hemisphere sees an object that the right hemisphere does not see, based on the behaviour of the human being. Here, 'the left hemisphere' and 'the right hemisphere' cannot plausibly be synecdoches for the whole human being, since then the resulting claim would be a contradiction, namely that the human being sees and does not see the object. But Schechter shows that a synecdochical interpretation of these locutions is still possible, though one in which 'the left hemisphere' is a synecdoche for the whole human being minus the right hemisphere, and 'the right hemisphere' is a synecdoche for the whole human being minus the left hemisphere (Schechter 2018: 82-83). Thanks to an anonymous referee for pointing this out.
} 
Finally, even though the Synecdoche View is restricted to ascriptions of psychological predicates to the brain made on grounds that are simultaneously grounds for ascribing those predicates to the human being, it is not restricted to ascriptions of psychological predicates to the brain made on the basis of the behaviour of the human being. Human beings can be ascribed psychological predicates on the basis of things that are not human behaviour, such as neural activity or computational simulations of neural activity. For example, it is common to ascribe psychological states to someone who is paralysed (e.g. someone with locked-in syndrome) on the basis of neural activity. So there may be cases in which brain activity is a ground for ascribing psychological predicates to both the brain and the human being. And in these cases, the ascription of psychological predicates to the brain can be accommodated by the Synecdoche View, since this view applies to any ascription of psychological predicates to the brain made on grounds that are simultaneously grounds for ascribing the same predicates to the human being - regardless of what those grounds are. What it does not apply to are ascriptions of psychological predicates to the brain made on grounds that are uniquely grounds for ascribing such predicates to the brain. ${ }^{10}$

\section{The Synecdoche View vs. the Nonsense View and the Literal View}

Unlike the Nonsense View and the Literal View, the Synecdoche View is agnostic about (P1) and (P2) of the Nonsense Argument, which say that it only makes sense to ascribe psychological predicates literally to an entity that (under normal circumstances) has the behaviour of an animal or human being. In other words, the Synecdoche View is agnostic about whether brain behaviour is a legitimate ground for ascribing psychological predicates to the brain - in a literal sense of 'brain'. The Synecdoche View is compatible with both Bennett and Hacker's view that brain behaviour is not a legitimate ground, and Dennett, Crane and Figdor's view that brain behaviour is a legitimate ground. But to repeat, this question of whether brain behaviour is a legitimate ground for ascribing psychological predicates to the brain is not relevant to the

\footnotetext{
${ }_{10}$ We are now in a position to see why (as mentioned in footnote 1), Figdor's (2018) view is ambiguous and may not be a defence of the Literal View at all. On the one hand, Figdor explicitly opposes Bennett and Hacker's Nonsense View and says that there are similarities between brains and human beings captured in mathematical models that warrant ascriptions of psychological predicates to brains (Figdor 2018: 96). But on the other hand, none of the locutions Figdor discusses from the scientific literature involve ascriptions of psychological predicates to brains. Rather, they involve ascriptions of psychological predicates to other entities discussed in biology, such as neurons, bacteria, plants, fruit flies, infants and cells. For these locutions, ascriptions of psychological predicates are made on the basis of the behaviour of these entities, in which case 'neurons', 'bacteria', 'plants' etc. should plausibly be taken literally and refer to neurons, bacteria, and plants respectively. This suggests an alternative interpretation of Figdor's view, according to which her view is not a defence of what I have called the 'Literal View'. For on my use of the phrase, the 'Literal View' refers a view about the ascription of psychological predicates to the brain. But Figdor's view may be restricted to ascriptions of psychological predicates to other entities in biology - made on the basis of the behaviour of those entities - such as bacteria, plants, etc. Her discussion of Bennett and Hacker's Nonsense View then, is perhaps only meant to address a possible opponent to that view and is therefore not strictly relevant to the debate with which this paper is concerned.
} 
semantics of the controversial locutions of cognitive scientists, since these locutions are not based on the observation of (or a mathematical model of) brain behaviour.

But the Synecdoche View is not agnostic about (P3) of the Nonsense Argument - that cognitive scientists make literal ascriptions of psychological predicates to the brain. This is something the Synecdoche View rejects but which the Nonsense View and the Literal View accept. The Synecdoche View accepts the assumption that psychological predicates should be taken literally, but it rejects the assumption that the word 'brain' demands the same literal interpretation. It is a live possibility that cognitive scientists are not referring to brains, but rather human beings in the controversial locutions where 'brain' is a grammatical subject of psychological predicates.

Once we see that cognitive scientists' grounds for ascribing psychological predicates to the brain are simultaneously grounds for ascribing those predicates to the human being, the Synecdoche View becomes available as a compelling option that can take on board aspects of both the alternative views. It agrees with the Nonsense View, contra the Literal View, that the relevant discoveries of cognitive scientists do not give us reason to believe that brains possess psychological properties, or that scientists have discovered that psychological predicates extend more widely than often assumed. This is how the Synecdoche View, which is primarily an answer to a semantic question, has implications for metaphysics. Yet, it agrees with the Literal View, contra the Nonsense View, that cognitive scientists' locutions both report empirical truths and make good sense, which a charitable interpretation of the linguistic practice of a successful science should try to maintain.

The Synecdoche View offers a plausible semantics of the controversial locutions of cognitive scientists. But as I said above, I only defend this modest claim and not the further claim that it offers the best semantics. The reason is that, while there is reason to prefer the Synecdoche View over the Nonsense View, the Literal View and the Synecdoche View seem to be on par in the sense that there is no compelling reason to prefer one over the other. Let me elaborate.

Even if proponents of the Nonsense View tried to accommodate the fact that cognitive scientists' grounds for ascribing psychological predicates to the brain are simultaneously grounds for ascribing such predicates to human beings, the Synecdoche View would be more plausible. The main reason is that it is more charitable to say that cognitive scientists synecdochically ascribe psychological predicates to the brain, rather than saying that their claims make no sense or are false. The Synecdoche View can maintain that the locutions of cognitive scientists make good sense and express truths, which a charitable interpretation of the linguistic practice of a successful science should aim for. In this respect, it is more plausible than the Nonsense View.

Now let's consider the Literal View. Remember the first two assumptions identified in $\$ 4$ - that (1) we are human beings and not brains, and (2) we possess psychological properties. If the human being literally thinks, and the brain literally thinks, and the grounds for ascribing thinking to both are the same, then both of them think simultaneously. This is a consequence of the Literal View, once it accommodates the fact that cognitive scientists' grounds for asserting that the brain thinks are simultaneously grounds for asserting that the human being whose brain it is thinks. The 
Synecdoche View, by contrast, only commits to one thinker in the literal sense, namely the human being. Olson has argued that it is implausible to hold that both the brain and the human being think simultaneously, because he thinks it follows that I cannot know who I am. His argument is the following. Given that the brain thinks when and only when the human being thinks, and what and only what the human being thinks, both the brain and the human being think they are me. The brain thinks it falsely and the human being thinks it truly, because by assumption I am the human being. But I cannot know whether I am the human being or the brain inside me that falsely thinks it is me (Olson 2007: 80-81, 215-216, Olson 2015: 46-47).

Perhaps proponents of the Literal View agree that we do not know who we are but deny that this is a problem. However, they need not. Parfit (2012: 20-22) points out an assumption that can be questioned in Olson's argument, namely that first person pronouns ('I', 'me') always refer to the thinker of a first person thought. ${ }^{11} \mathrm{~A}$ proponent of the Literal View can claim that those pronouns only refer to me, i.e. the human being, and not my brain, whenever my brain thinks thoughts involving first person pronouns. Consequently, it is not the case that my brain mistakenly thinks that it is me. The brain does not think that it is anything, because first person pronouns involved in its thoughts refer to me - the human being. So I can know who I am, namely the human being. And there is no question of the brain knowing who it is, for when it thinks that I am me, it does not (falsely) think that it is me, it (truly) thinks that I - the human being - am me - the human being. Thus, defenders of the Literal View can maintain that I know who I am.

To my knowledge, there is no further reason to prefer the Synecdoche over the Literal View. But is there any reason to prefer the Literal View? In discussion I have come across three arguments but neither of them are convincing.

The first argument is that that the brain actually possesses psychological properties: if the brain thinks, then it is plausible to interpret 'The brain thinks' literally. But it is not clear why we should believe that the brain possesses psychological properties independent of the Literal View. If it is true that the brain possesses psychological properties, then that is not something we know a priori, but presumably something we know from science. How can we know whether science teaches us that brains possess psychological properties? We can begin by looking at what scientists say. And some cognitive scientists do ascribe psychological predicates to the brain and say things like 'The brain thinks'. But this is only evidence that the brain possesses psychological properties if those locutions should be interpreted literally. I have argued above that locutions like those documented and discussed in the literature can plausibly be interpreted non-literally and that proponents of the Literal View have yet to provide evidence of other locutions that cannot plausibly be interpreted this way. The claim that the brain possesses psychological properties cannot be the support for the Literal View if the Literal View is the support for the claim that the brain possesses psychological properties, since that would be circular.

\footnotetext{
11 Parfit (2012) thinks that we are brains, so I have rephrased his argument in a way consistent with the assumption that we are human beings.
} 
The second argument to prefer the Literal View is that interpreting the locutions of scientists literally is the default interpretation (cf. Figdor 2018: 87-88). But it is not clear why this is so. Scientific language often involves figurative elements, such as metaphors (Boyd 1979). So even though the default may be to interpret the locutions of scientists as making sense rather than being nonsensical, and reporting empirical truths rather than falsehoods, it is not clear why a literal interpretation should be the default interpretation.

The third argument to prefer the Literal View is that locutions with relevant similarities to the controversial locutions of cognitive scientists are literal. We say both 'the human being kicks' and 'the foot kicks' because the foot is functionally relevant to kicking, and the grounds for ascribing kicking to the foot are simultaneously grounds for ascribing kicking to the human being. Similarly, we say both 'the eye sees' and 'the human being sees' because the eye is functionally relevant to seeing, and the grounds for ascribing seeing to the eye are simultaneously grounds for ascribing seeing to the human being. Given that these locutions are all literal and have several relevant similarities to the controversial locutions of cognitive scientists, it is plausible to interpret the latter locutions literally. But a defender of the Synecdoche View need not accept that locutions like 'the foot kicks' and 'the eye sees' are literal. These locutions have a well-established use but that does not imply that they are literal. They may be figurative, but in lack of a commonly accepted criterion for literalness or figurativeness it is not clear how to determine the issue.

It therefore seems to me that the Literal View and the Synecdoche View are on par and that there is currently no compelling reason to prefer one rather than the other. Hence my modest claim that the Synecdoche View is a plausible (not the 'best') alternative.

\section{Conclusion}

It is a live possibility that cognitive scientists' ascriptions of psychological predicates to the brain should be understood synecdochically and report the psychological properties of human beings - at least those ascriptions made on grounds that are simultaneously grounds for ascribing psychological predicates to human beings. This claim rests on the assumptions that (1) we are human beings and not brains, (2) we possess psychological properties, and (3) cognitive scientists use psychological predicates with a literal sense. If one rejects (1) or (2), perhaps the best interpretation of the controversial locutions is a literal interpretation and that ascriptions of psychological predicates to human beings should be interpreted synecdochically instead - with 'human being' referring to the brain. If one rejects (3), perhaps the best interpretation is one according to which cognitive scientists' ascriptions of psychological predicates to the brain is just a colourful way of talking about neural activity. I have not addressed these alternative views because my aim was to argue that the Synecdoche View is a plausible view on the assumption that (1)-(3) is true. 
Even though I do not make the further claim that the Synecdoche View is the best view, the claim that it is a plausible view is still an interesting result, both for semantics and metaphysics. One consequence is that, contrary to both the Nonsense View and the Literal View, there is no reason to take the controversial locutions literally. A second consequence is that, contrary to what e.g. Figdor (2018: 5-6, 61) claims, there is no reason to believe that the controversial locutions indicate empirical support for the claim that brains possess psychological properties, or that scientists have discovered that psychological predicates extend to a wider range of entities than often assumed. These are substantive and controversial consequences, which indicate the significance of the Synecdoche View.

Acknowledgement Thanks to Arif Ahmed, Anna Alexandrova, Lukas Beck, Luke Cash, Tim Crane, Ståle Finke, Ludvig Fæhn Fuglestvedt, Marta Halina, Oscar Westerblad, and an anonymous referee for comments on earlier versions of this paper. Thanks also to members of Oslo Mind Group at the University of Oslo and Peter Hacker for helpful discussion, and to Aker Scholarship for financial support.

Availability of data and material Not applicable

Authors' contributions Not applicable

Funding information Aker Scholarship

\section{Declarations}

\section{Conflicts of interest/Competing interests None}

Code availability Not applicable

Open Access This article is licensed under a Creative Commons Attribution 4.0 International License, which permits use, sharing, adaptation, distribution and reproduction in any medium or format, as long as you give appropriate credit to the original author(s) and the source, provide a link to the Creative Commons licence, and indicate if changes were made. The images or other third party material in this article are included in the article's Creative Commons licence, unless indicated otherwise in a credit line to the material. If material is not included in the article's Creative Commons licence and your intended use is not permitted by statutory regulation or exceeds the permitted use, you will need to obtain permission directly from the copyright holder. To view a copy of this licence, visit http://creativecommons.org/licen ses/by/4.0/.

\section{References}

Baker G. P. and Hacker P. M. S. 2005 Wittgenstein: Understanding and Meaning. $2^{\text {nd }}$ ed. extensively revised by P. M. S. Hacker. Oxford: Blackwell.

Bennett M. R. 2007 Philosophy and Neuroscience. In Bennett M. R. et al. Neuroscience and Philosophy. New York: Columbia University Press.

Bennett, M.R., and P.M.S. Hacker. 2003. Philosophical Foundations of Neuroscience. Oxford: Blackwell.

Bennett M. R. and Hacker P. M. S. 2007 The Conceptual Presuppositions of Cognitive Neuroscience: A Reply to Critics. In Bennett M. R. et al. Neuroscience and Philosophy. New York: Columbia University Press.

Boyd R. 1979 Metaphor and Theory Change: What is 'Metaphor' a Metaphor For? In Ortony A. (ed.) Metaphor and Thought. Cambridge: Cambridge University Press. 
Crane, T. 2015. The Mental States of Persons and their Brains. Royal Institute of Philosophy Supplement 76.

Crick, F. 1994. The Astonishing Hypothesis: the Scientific Search for the Soul. New York: Charles Scribner's Sons.

Damasio, A. 1996. Descartes' Error - Emotion, Reason and the Human Brain. London: Papermac.

Dennett, D. 1975. Three Kinds of Intentional Psychology. In Reduction, Time, and Reality: Studies in the Philosophy of the Natural Sciences, ed. R. Healy. Cambridge: Cambridge University Press.

Dennett D. 2007 Philosophy as Naïve Anthropology: Comment on Bennett and Hacker. In Bennett M. R. et al. Neuroscience and Philosophy. New York: Columbia University Press.

Drayson, Z. 2014. The Personal/Subpersonal Distinction. Philosophy Compass 9.

Figdor, C. 2018. Pieces of Mind. The Proper Domain of Psychological Predicates. Oxford: Oxford University Press.

Frith, C. 2007. Making Up the Mind: How the Brain Creates Our Mental World. Oxford: Blackwell.

Gazzaniga M. 1995 Principles of Human Brain Organization Derived from Split-Brain Studies. Neuron 14.

Hacker, P.M.S. 2013. The Intellectual Powers: A Study of Human Nature. Oxford: Wiley Blackwell.

Hacker, P.M.S. 1990. Wittgenstein - Meaning and Mind. An Analytic Commentary on the Philosophical Investigations. Vol. 3. Oxford: Blackwell.

Körding, K.P., and D.M. Wolpert. 2004. Bayesian Integration in Sensorimotor Learning. Nature 427.

Lakoff, G., and M. Johnson. 1980. Metaphors We Live By. Chicago: The University of Chicago Press.

LeDoux, J. 1998. The Emotional Brain. London: Phoenix.

Machamer P. and Sytsma J. 2009 Philosophy and the Brain Sciences. Iris. European Journal of Philosophy and Public Debate 1.

Olson, E. 2007. What Are We? Oxford: Oxford University Press.

Olson, E. 2015. On Parfit's View That We Are Not Human Beings. Royal Institute of Philosophy Supplement 76.

Parfit D. 2012 We Are Not Human Beings. Philosophy 87.

Schechter, E. 2018. Self-Consciousness and "Split" Brains: The Minds' I. Oxford: Oxford University Press.

Searle J. 2007 Putting Consciousness Back in the Brain: Reply to Bennett and Hacker, Philosophical Foundations of Neuroscience. In Bennett M. R. et al. Neuroscience and Philosophy. New York: Columbia University Press.

Smythies J. 2009 Philosophy, Perception, and Neuroscience. Perception 38.

Sperry R. 1974 Lateral Specialization in the Surgically Separated Hemispheres. In Schmitt F. O and Worden F. G. (eds.) The Neurosciences Third Study Programme. Cambridge, MA: MIT Press.

Wilson D. and Sperber D. 2002 Truthfulness and Relevance. Mind 111.

Publisher's Note Springer Nature remains neutral with regard to jurisdictional claims in published maps and institutional affiliations. 